Methods: The analysis included 80 APS patients ( $\leq 50$ years old) who presented a previous stroke event (patients who experienced cerebral venous sinus thrombosis were not included in the analysis). Clinical and laboratory data were retrospectively collected. Treatment was based on phisician's opinion according to clinical settings. The aGAPSS was calculated for each patient by adding the points corresponding to the risk factors, based on a linear transformation derived from the $\beta$ regression coefficient as follows: 3 for hyperlipidaemia, 1 for arterial hypertension, 5 for aCL $\mathrm{IgG} / \mathrm{lgM}, 4$ for antiß2glycoprotein I IgG/lgM and 4 for LA. Relapse was defined as the recurrence of thrombotic event and/or progression of known ischaemic lesions detected with MRI.

Results: Results pointed out that patients with relapse of thrombotic events and/or progression of known ischaemic lesions were 39 out of $80(48.7 \%)$ and patients without relapse were 41 out of $80(51.3 \%)$. Significantly higher aGAPSS values were observed in relapse group when compared to the non-relapse group [mean aGAPSS 9.08 (S.D. 4.7) Vs. mean aGAPSS 7.22 (S.D. 3.3); T test: $p<0.05]$. Distribution of aGAPPS values among the two groups is illustrated in Graph 1.

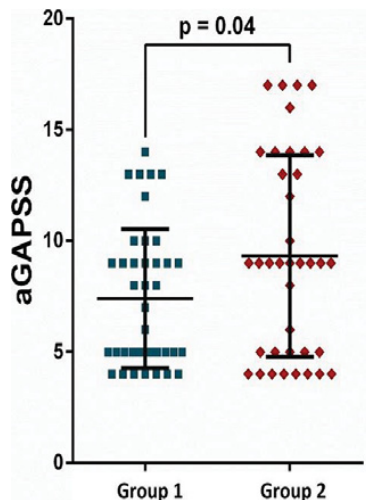

Graph 1.: Distribution of aGAPPS values among the two groups; Group 1: patients without relapse of thrombotic events and/or progression of known ischaemic lesions detected with MRI; Group 2: patients who presented relapse of thrombotic events and/or progression of ischaemic lesions.

Conclusions: Our analysis suggests that aGAPSS could represent an effective tool to stratify the risk of relapse of thrombosis and/or progression of ischaemic lesions in young APS patients with clinical history of stroke. These data could also aid developing different therapeutic approaches, especially for patients at higher risk of relapse.

References:

[1] Tektonidou MG, Varsou N, Kotoulas G, Antoniou A, Moutsopoulos HM. Cognitive deficits in patients with antiphospholipid syndrome: association with clinical, laboratory, and brain magnetic resonance imaging findings. Arch Intern Med 2006;166(20):2278-84.

[2] Meroni PL, Borghi MO, Raschi E, Tedesco F. Pathogenesis of antiphospholipid syndrome: understanding the antibodies. Nat Rev Rheumatol. 2011;7:330-9.

[3] Sciascia S, Sanna G, Murru V, Roccatello D, Khamashta MA, Bertolaccini ML. GAPSS: The Global Anti-Phospholipid Syndrome Score. Rheumatology (Oxford) 2013; 52:1397-403.

Acknowledgements: None.

Disclosure of Interest: None declared

DOI: 10.1136/annrheumdis-2017-eular.6891

\section{AB1131 RISK OF DEVELOPING ATHEROSCLEROSIS IN PATIENTS WITH RHEUMATOID ARTHRITIS}

I. Starodubtseva. Internal Diseases, Nn Burdenko Voronezh State Medical University, Voronezh, Russian Federation

Background: Patients with chronic inflammatory diseases have an increased risk of developing atherosclerosis. Pro-inflammatory cytokines such as interleukin-(IL) 1 beta and tumor necrosis factor-(TNF-)alpha, C-reactive protein (CRP) influence on the progression and development of both rheumatoid arthritis (RA) and atherosclerosis.

Methods: 620 patients with RA (diagnosis according to ACR /EULAR) from the rheumatology in-patient clinic with the mean age of $43.4 \pm 10 ; 95.4 \%$ ACCP positive patients, activity on DAS (Disease Activity Score) $28 \mathrm{II}$, III; 85.4\% female with the disease duration for about 3-15 years were enrolled in the trial. We assessed the level of IL-1 with the use of ELISA.

Results: The constructed model surfaces indicated the interdependence of IL-1, the activity of DAS 28 and the level of LDH in RA patients. The correlative and regressive analysis of the results showed the statistically significant correlation of TG, LDG and markers of inflammation IL-1, DAS 28: $p=0.627(p<0.01), p=0.527$ $(p<0.01), p=0.712(p<0.01), p=0.776 \quad(p<0.01)$ accordingly. The correlation coefficient between hs-CPR and the indicators of the lipid profile revealed similar interconnections.

Conclusions: According to the results of modeling, disease activity on DAS and markers of inflammation (IL-1 and hs-CRP) as a markers of the severity of inflammatory process in RA patients are risk factors for developing atherosclerosis. The analysis of inflammation indicators in RA patients allows to assess the risk of developing and progressing atherosclerosis. The data enables to select the best possible personified therapy for such patients at the early stage of the disease.

Disclosure of Interest: None declared

DOI: 10.1136/annrheumdis-2017-eular.1677

\section{AB1132 HIGHER RATES OF OBESITY AND ASSOCIATIONS WITH POORER CLINICAL STATUS IN PATIENTS WITH RA, OA AND SLE: A CROSS-SECTIONAL STUDY FROM ROUTINE CARE}

I. Castrejon, N. Shakoor, J.A. Block, T. Pincus. Rheumatology, Rush University Medical Center, Chicago, United States

Background: Obesity is a risk factor for many chronic rheumatic diseases In rheumatoid arthritis (RA), obesity is associated with increased comorbidities, higher medical costs, disease activity, and poorer physical function ${ }^{1}$. In OA, obesity is a risk factor for both incidence and progression, and has a negative impact on outcomes ${ }^{2}$. In systemic lupus erythematous (SLE), obesity is associated with more severe renal involvement, lower quality of life, and increased cardiovascular risk $^{3}$

Objectives: To assess associations of obesity with patient self-report multidimensional health assessment questionnaire (MDHAQ) scores and physician global assessments in patients with RA, OA and SLE seen in routine care.

Methods: All patients at one academic center complete a MDHAQ, which includes a $0-10$ scale for physical function (FN), 0-10 visual analogue scales (VAS) for pain (PN) and patient global assessment (PATGL), compiled into a 0-30 RAPID3, as well as scales for fatigue, depression, and demographic data. Physicians complete a VAS for patient global (DOCGL). Body Mass Index (BMI) was calculated from the medical record as weight $(\mathrm{kg}) /$ height (meters) ${ }^{2}$. Patients were classified by BMI as normal (18.5-25), overweight (25-30), or obese $(>30)$ according to the WHO guidelines. Demographic and clinical MDHAQ data were compared in the 3 diagnostic groups according to BMI groups using ANOVA and chi-square tests.

Results: 396 patients with RA, 425 with OA, and 306 with SLE were studied. Obesity was reported by $40 \%$ of RA and SLE patients, and $59 \%$ of OA patients, a higher percentage than matched individuals in the general population in the same region (30.8\%). Obesity was higher in African-American patients $(48 \%$ in RA, $70 \%$ in OA, and $53 \%$ in SLE). Education level, gender, and age did not differ significantly across the groups. Obesity was associated with poorer physical function, poorer patient global and higher pain in all 3 diagnostic groups, with higher depression scores in OA and SLE (Table). DOCGL was significantly higher only in OA (data not shown).

Table 1. MDHAQ scores and physician global assessment according to BMI groups

\begin{tabular}{lccc}
\hline MDHAQ scores & Normal (BMl=18.5-25) & Overweight (BMI=25-30) & Obesity (BMI>30) \\
\hline RA (N=381) & $110(29 \%)$ & $112(30 \%)$ & $154(40 \%)$ \\
Function (0-10) & $2.1(2.2)$ & $2.4(2.2)$ & $2.9(2.0)^{\star}$ \\
Pain (0-10) & $4.4(2.8)$ & $4.6(3.0)$ & $5.1(3.1)^{\star}$ \\
Fatigue (0-10) & $3.4(2.9)$ & $3.6(3.1)$ & $4.5(3.2)^{\star}$ \\
PATGL (0-10) & $3.8(2.7)$ & $4.1(3.1)$ & $4.8(2.8)^{\star}$ \\
Depression (0-3.3) & $0.5(0.7)$ & $0.5(0.7)$ & $0.6(0.8)$ \\
OA (N=420) & $60(14 \%)$ & $102(24 \%)$ & $247(59 \%)$ \\
Function (0-10) & $1.7(1.5)$ & $2.6(1.8)$ & $3.2(2.0)^{\star}$ \\
Pain (0-10) & $5.1(2.9)$ & $6.7(2.5)$ & $6.6(2.6)^{\star}$ \\
Fatigue (0-10) & $3.4(2.9)$ & $4.3(2.9)$ & $5.3(3.1)^{\star}$ \\
PATGL (0-10) & $4.5(3.1)$ & $5.7(2.5)$ & $5.9(2.7)^{\star}$ \\
Depression (0-3.3) & $0.4(0.6)$ & $0.6(0.8)$ & $0.7(0.8)^{\star}$ \\
SLE (N=299) & $84(28 \%)$ & $85(28 \%)$ & $121(40 \%)$ \\
Function (0-10) & $1.4(1.5)$ & $1.2(1.6)$ & $2.3(2.1)^{\star}$ \\
Pain (0-10) & $3.5(3.2)$ & $3.9(3.1)$ & $5.2(3.3)^{\star}$ \\
Fatigue (0-10) & $4.2(3.3)$ & $4.2(3.4)$ & $5.1(3.2)$ \\
PATGL (0-10) & $3.6(2.9)$ & $3.9(3.1)$ & $4.6(3.2)^{\star}$ \\
Depression (0-3.3) & $0.4(0.6)$ & $0.4(0.6)$ & $0.7(0.8)^{\star}$ \\
\hline
\end{tabular}

${ }^{*} \mathrm{p}<0.01$.

Conclusions: Obesity is more prevalent in patients with rheumatic diseases compared with the general population. Obese patients had poorer status on most MDHAQ scores, particularly physical function and pain. Obesity is an important comorbidity in patients with rheumatic diseases.

References:

[1] J Rheumatol 2015, 42(12):2261-2269.

[2] Obes Rev 2014, 15(7):578-586.

[3] Autoimmunity Reviews 2014;13: 981-1000.

Disclosure of Interest: I. Castrejon: None declared, N. Shakoor: None declared, J. Block: None declared, T. Pincus Shareholder of: Health Report Services, Inc DOI: 10.1136/annrheumdis-2017-eular.3812 